06,11

\title{
Исследование диэлектрического отклика, проводимости и тока термостимулированной деполяризации в релаксорном сегнетоэлектрике $\mathrm{PbNi}_{1 / 3} \mathrm{Nb}_{2 / 3} \mathrm{O}_{3}$
}

\author{
() А.Д. Полушина, Е.Д. Обозова, В.Г. Залесский, Т.А. Смирнова, С.Г. Лушников \\ Физико-технический институт им. А.Ф. Иоффре РАН, \\ Санкт-Петербург, Россия \\ E-mail: nsh@mail.ioffe.ru
}

Поступила в Редакцию 18 апреля 2021 г.

В окончательной редакции 18 апреля 2021 г.

Принята к публикации 19 апреля 2021 г.

Представлены результаты исследования температурных зависимостей диэлектрического отклика и проводимости на постоянном и переменном токе в монокристаллах релаксорного сегнетоэлектрика $\mathrm{PbNi}_{1 / 3} \mathrm{Nb}_{2 / 3} \mathrm{O}_{3}$ $(\mathrm{PNN})$ в широком интервале частот $(10-100 \mathrm{kHz})$ и температур $(80-750 \mathrm{~K})$. Аномалии диэлектрического отклика в виде широких частотно-зависимых максимумов наблюдаются в окрестности 153 и $730 \mathrm{~K}$. Установлен термоактивационный характер проводимости на постоянном токе и определены энергии активации $E_{a}=770 \mathrm{meV}$ при $T>310 \mathrm{~K}$ и $E_{a}=23 \mathrm{meV}$ при $T<310 \mathrm{~K}$. Результаты анализа проводимости дают основание предполагать существование локальной проводимости в области низких температур. Показано, что характер проводимости меняется от локальной к объемной при увеличении температуры, начиная с $350 \mathrm{~K}$. Методом измерения тока термостимулированной деполяризации определена температурная область существования остаточной поляризации (наблюдается при $T<130 \mathrm{~K}$ ).

Ключевые слова: сегнетоэлекрики-релаксоры, мультиферроики, поляризация, диэлектрическая проницаемость, проводимость.

DOI: $10.21883 /$ FTT.2021.09.51314.088

\section{1. Введение}

Многочисленное семейство кристаллов со структурой перовскита $A B^{\prime} B^{\prime \prime} \mathrm{O}_{3}(A=\mathrm{Pb})$ со сложным замещением четырехвалентного иона в $B$-позиции двумя ионами разной валентности проявляют уникальные физические свойства релаксорных сегнетоэлектриков [1]. Для релаксорных сегнетоэлектриков (далее - релаксоры), например для $\mathrm{PbMg}_{1 / 3} \mathrm{Nb}_{2 / 3} \mathrm{O}_{3}$ (PMN), характерна температурная зависимость диэлектрической проницаемости в виде широких частотно-зависимых максимумов, не связанных, в общем случае, со структурным фазовым переходом. Область температур, в которой наблюдаются широкие температурные, зависимые от частоты аномалии диэлектрического отклика получила название области размытого фазового перехода [1]. Соединения этого семейства, а также твердые растворы на их основе, обладают гигантскими диэлектрическими и пьезоэлектрическими коэффициентами, что широко используется в промышленности. В семействе релаксоров можно выделить отдельную группу кристаллов, у которых один из ионов в $B$-позиции является магнитоактивным. Сочетание магнитных свойств ферро- и антиферромагнетиков и полярных свойств релаксоров позволяет рассматривать эту группу кристаллов в качестве перспективных мультифункциональных материалов, получивших название мультиферроиков-релаксоров [2,3].
Потенциальным кандидатом в мультиферроики-релаксоры можно рассматривать малоизученный кристалл $\mathrm{PbNi}_{1 / 3} \mathrm{Nb}_{2 / 3} \mathrm{O}_{3}$ (PNN). Кристалл обладает кубической симметрией $P m 3 m$ с параметром решетки $a=4.04 \AA$ [4]. Температурная зависимость диэлектрической проницаемости в монокристаллических и керамических образцах PNN сопровождается аномалиями в виде широких частотно-зависимых максимумов в окрестности $150 \mathrm{~K}$, характерных для релаксоров. Согласно данным первой публикации по PNN [5] величина максимумов достигает $\varepsilon_{m}(1 \mathrm{kHz})=3000$ при $T_{m}=148 \mathrm{~K}$. В более поздних работах можно отметить некоторый разброс данных, где соответствующие величины варьируются в следующих пределах: $\varepsilon_{m}=2400-3800, T_{m}=120-153 \mathrm{~K}[4,6,7]$. Можно предположить, что некотоый разброс значений $\varepsilon_{m}$ и $T_{m}$ связан с особенностями технологии роста кристаллов. В некоторых работах [7] высказывалось предположение о существовании структурного фазового перехода в PNN из параэлектрической кубической фазы в сегнетоэлектрическую ромбоэдрическую $P 31 \mathrm{~m}$ при $153 \mathrm{~K}$. На это указывало, в том числе, и появление поперечных акустических фононов в бриллюэновских спектрах рассеяния света при температурах ниже $150 \mathrm{~K}$, которые запрещены правилами отбора кубического кристалла, в используемой в работе [7] геометрии рассеяния. Рамановские исследования кристаллов PNN показали отсутствие ярко выраженной эволюции оптических мод в окрестности размытого фазового перехода 
в окрестности $150 \mathrm{~K}[8,9]$, что характерно для релаксоров [10]. В настоящий момент отсутствуют результаты структурных исследований кристалла PNN, что затрудняет однозначную интерпретацию литературных данных, в том числе и возможность существования структурного фазового перехода при $T<150 \mathrm{~K}$. Исследования магнитных свойств PNN показали сложную температурную зависимость магнитной восприимчивости керамических образцов, которая ниже $100 \mathrm{~K}$ достаточно хорошо описывается моделью, учитывающей вклады, как от отдельных ионов $\mathrm{Ni}^{2+}$, так и от цепочек $\mathrm{Ni}-\mathrm{O}-\mathrm{Ni}$ c антиферромагнитным упорядочением [11].

Изучение полярного состояния кристаллов PNN проводилось по результатам исследования петель диэлектрического гистерезиса [4]. Для монокристаллов PNN $P$-E-петли, близкие к насыщению, наблюдались только при $T \sim 77 \mathrm{~K}$, в поле $200 \mathrm{kV} / \mathrm{cm}$, достигающего уровня пробоя. Авторы работы [4] не исключали того, что полученные петли гистерезиса могут быть обусловлены нелинейным диэлектрическим откликом. Тем более, что при охлаждении кристалла до гелиевых температур зависимость поляризации от поля становилась линейной. Таким образом, вопрос о температурной области существования полярного состояния в PNN остается открытым. Для исследования поляризации и определения интервала температур ее существования, в мультиферроиках с относительно высокой проводимостью часто применяется метод термостимулированной деполяризации $[12,13]$, который мы использовали в данной работе. При этом для корректного измерения тока деполяризации необходимы данные о механизмах проводимости кристалла PNN и о наличии аномалий проводимости в исследуемом диапазоне температур. Это стимулировало нас в проведении исследований проводимости в широком интервале частот и температур в кристалле PNN.

\section{2. Методика и материалы}

Монокристаллы PNN, прозрачные, желто-зеленого цвета, были выращены методом спонтанной кристаллизации. Химический состав кристаллов был подтвержден рентгенофлуоресцентным методом. После ориентации образцов PNN с помощью рентгенодифракционной установки ДРОН-3 из кристаллов были вырезаны пластины толщиной $0.27 \mathrm{~mm}$ и площадью наибольшей грани $2 \mathrm{~mm}^{2}$ в плоскости (001). Поверхности, отполированные до оптического качества, были покрыты золотыми электродами методом термического напыления. Измерения диэлектрической проницаемости и тангенса потерь проводились импедансметром Good Will LCR-819 в поле амплитудой $1 \mathrm{~V}$ и частотой от $10 \mathrm{~Hz}$ до $100 \mathrm{kHz}$, в интервале температур $77-800 \mathrm{~K}$. Скорость нагрева и охлаждения $1-3 \mathrm{~K} / \mathrm{min}$ (вблизи максимумов скорость снижалась до $0.5 \mathrm{~K} / \mathrm{min}$ ). Проводимость на постоянном токе измерялась тераомметром Е6-13А в том же интервале температур.
Ток термостимулированной деполяризации измерялся электрометрическим усилителем У119 с чувствительностью до $0.1 \mathrm{pA}$. Эксперимент проводился по стандартной схеме измерений в два этапа [13]. На первом этапе образец охлаждался от 400 до $77 \mathrm{~K}$ во внешнем поляризующем поле $30 \mathrm{~V}(1.1 \mathrm{kV} / \mathrm{cm})$. Затем, при температуре $77 \mathrm{~K}$ образец выдерживался в короткозамкнутом состоянии в течение $5 \mathrm{~min}$ для снятия приповерхностного заряда. На втором, основном этапе, проводилось измерение тока деполяризации в режиме нагревания с постоянной скоростью $V_{T}=5 \mathrm{~K} / \mathrm{min}$. Эксперимент повторялся для поля другой полярности.

\section{3. Экспериментальные результаты и обсуждение}

\section{1. Диэлектрический отклик и проводимость в PNN}

Температурная зависимость $\varepsilon^{\prime}(T)$, действительной части комплексной диэлектрической проницаемости $\varepsilon^{*}(T)=\varepsilon^{\prime}(T)-i \varepsilon^{\prime \prime}(T)$ приведена на рис. $1, a$. Широкий максимум диэлектрической проницаемости на частоте $1 \mathrm{kHz}$ достигает величины $\varepsilon_{\max }^{\prime}=5500$ при температуpe $T_{\max }=153 \mathrm{~K}$ (для сравнения, в модельном релакcope PMN: $\varepsilon_{\max }^{\prime}=11400$ и $T_{\max }=265 \mathrm{~K}$ ). Положение максимума зависит от частоты измерительного сигнала, в интервале частот $100 \mathrm{~Hz}-100 \mathrm{kHz}$ наблюдается смещение $T_{\max }$ на $17 \mathrm{~K}$ (в $\mathrm{PMN}-18 \mathrm{~K}$ ) в сторону более высоких температур. Ширина максимума на уровне половины его высоты значительно больше, чем в PMN и составляет $145 \mathrm{~K}$ ( $80 \mathrm{~K}$ для $\mathrm{PMN})$. На врезке к рис. 1, $a$ показана температурная зависимость обратной величины диэлектрической проницаемости с целью проверки соответствия закону Кюри-Вейсса $\varepsilon^{\prime}(T)=C_{\mathrm{CW}} /\left(T-T_{\mathrm{CW}}\right)$. Со стороны низких температур получен линейный участок со следующими параметрами: $T_{\mathrm{CW}}=150 \mathrm{~K}, C_{\mathrm{CW}}=7.5 \cdot 10^{4} \mathrm{~K}$, в то время как со стороны высоких температур можно выделить два линейных участка со следующими значениями величин: $T_{\mathrm{CW}}=150 \mathrm{~K}, C_{\mathrm{CW}}=1.0 \cdot 10^{6} \mathrm{~K}$ и $T_{\mathrm{CW}}=210 \mathrm{~K}$, $C_{\mathrm{CW}}=2.1 \cdot 10^{5} \mathrm{~K}$. Наличие трех линейных участков в зависимости $1 / \varepsilon^{\prime}(T)$, отклонение от закона ,двойки“ и более широкий максимум по сравнению с PMN, возможно, обусловлены наличием еще одной, слабой аномалии в области температур 200-210 K [14]. В высокотемпературной области выявлена аномалия в виде размытого максимума при $730 \mathrm{~K}$, который с увеличением частоты смещается в область более высоких температур, а его амплитуда уменьшается (рис. $1, a$ ).

На температурной зависимости мнимой части диэлектрического отклика $\varepsilon^{\prime \prime}(T)$ (рис. $\left.1, b\right)$ частотно-зависимый максимум наблюдается в окрестности температуры $138 \mathrm{~K}(1 \mathrm{kHz})$. Ширина максимумов на уровне половины высоты примерно равна $25 \mathrm{~K}$, как и в PMN. Также наблюдается характерное для релаксоров смещение температуры максимума $T_{m}$ в сторону больших 

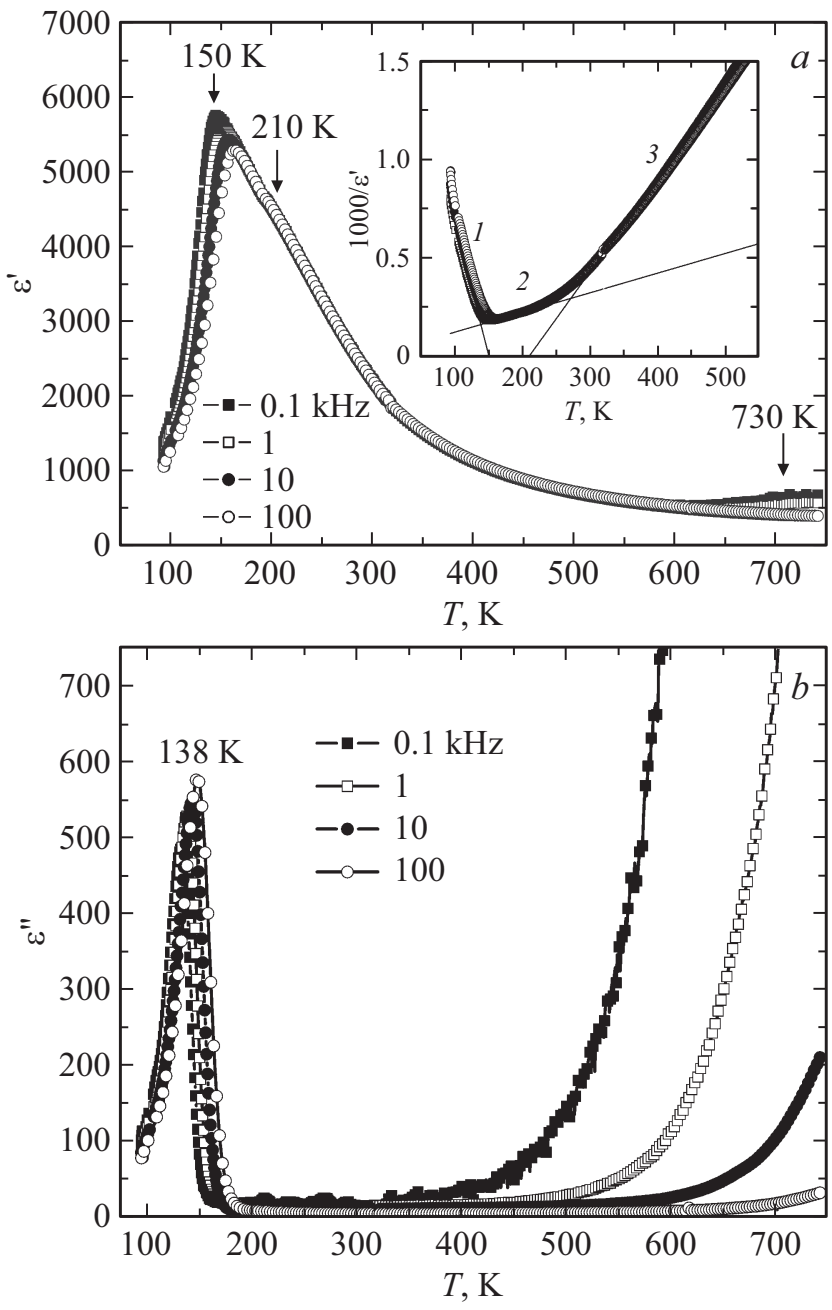

Рис. 1. Температурная зависимость $a$ ) действительной и $b)$ мнимой частей диэлектрического отклика в монокристалле PNN при разных частотах. На вставке к рис. 1, $a$ показана температурная зависимость обратной величины диэлектрической проницаемости, линии показывают аппроксимацию зависимости с помощью закона Кюри-Вейсса.

температур с увеличением частоты. В районе комнатной температуры величина диэлектрических потерь мала, $\operatorname{tg} \delta<0.02$, что связано с малой удельной проводимостью кристаллов PNN. При температуре выше $550 \mathrm{~K}$ наблюдаются характерные возрастание диэлектрических потерь и рост дисперсии, которые обусловлены ростом проводимости кристалла с увеличением температуры. Такое высокотемпературное поведение $\varepsilon^{\prime \prime}$ маскирует аномалию в окрестности $730 \mathrm{~K}$, наблюдаемую в температурных зависимостях диэлектрической проницаемости (см. рис. 1, $a$ и $b$ ).

B кристалле PNN с большой величиной диэлектрической проницаемости существует зарядовое разупорядочение, поэтому важной характеристикой кристалла является температурно-частотная зависимость проводимости, приведенная на рис. 2. На рисунке хорошо видны частотно-зависимые максимумы проводимости в окрестности $138 \mathrm{~K}$, а при температуре выше $500 \mathrm{~K}$ наблюдается монотонное возрастание проводимости. Общая проводимость складывается из проводимости на постоянном и переменном токе $\sigma=\sigma_{\mathrm{dc}}+\sigma_{\mathrm{ac}}(\omega)$. Проводимость на постоянном токе $\sigma_{\mathrm{dc}}$ представлена на вставке к рис. 2. Из рисунка хорошо видно, что она имеет термоактивационный характер и следует закону Аррениуса $\sigma(T)=\sigma_{0} \exp \left(-E_{a} / k T\right)$, где $E_{a}-$ энергия активации, $k-$ постоянная Больцмана. На температурной зависимости $\sigma_{\mathrm{dc}}(T)$ можно выделить два участка: в интервале температур ниже $310 \mathrm{~K}$ величина удельной dc-проводимости составляет $10^{-11}-10^{-12} \Omega^{-1} \mathrm{~cm}^{-1}$, а энергия активации носителей $E_{a}$ оценивается величиной $23 \mathrm{meV}$. Выше температуры $310 \mathrm{~K}$ энергия активации возрастает на порядок, достигая величины $770 \mathrm{meV}$.

Проводимость $\sigma_{\mathrm{ac}}$ на высоких частотах обладает частотной дисперсией и обусловлена диэлектрическими потерями $\sigma_{\mathrm{ac}}(\omega)=\omega \varepsilon_{0} \varepsilon^{\prime \prime}(\omega)$. Как хорошо видно из рис. 2, чем выше частота, тем выше проводимость. Такая частотная дисперсия характерна для локальной проводимости внутри ограниченных областей кристалла с активационными барьерами на их границах [15]. Для анализа свойств таких областей представим локальную проводимость в относительном масштабе: $\sigma_{\mathrm{loc}}=\left(\sigma_{\mathrm{ac}}-\sigma_{\mathrm{dc}}\right) /\left(\sigma_{\mathrm{ac}}+\sigma_{\mathrm{dc}}\right)$. Температурная зависимость $\sigma_{\mathrm{loc}}(T)$ в таком представлении хорошо характеризует отношение локальной проводимости к сквозной проводимости и фиксирует интервалы температур существования упомянутых областей с локальной проводимостью [15].

На рис. 3 представлены температурные зависимости $\sigma_{\text {loc }}(T)$ на различных частотах относительно проводимости на самой низкой измеренной частоте. Из этих зависимостей хорошо виден интервал температур ниже $400 \mathrm{~K}$, в котором $\sigma_{\text {loc }}$ превышает сквозную проводи-

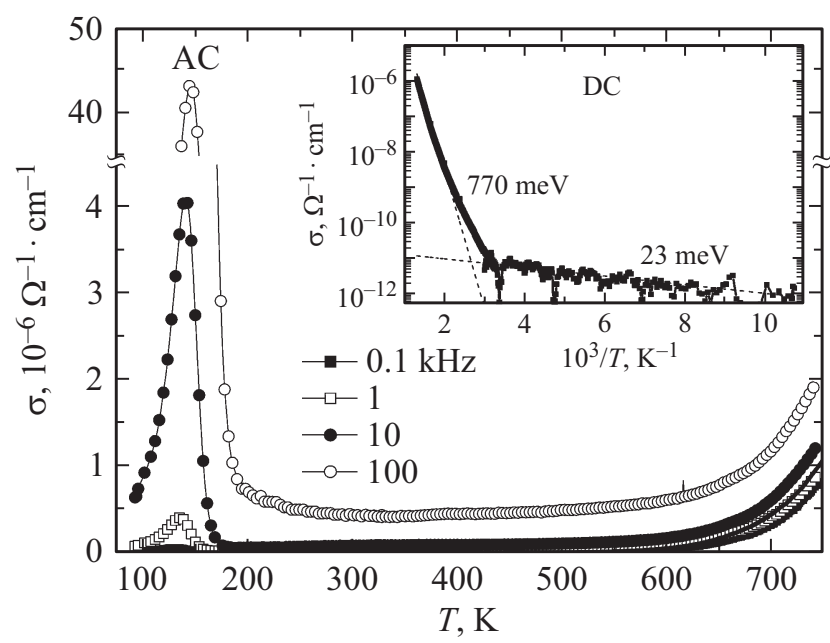

Рис. 2. Температурная зависимость проводимости в кристалле PNN на разных частотах. На вставке показана температурная зависимость проводимости на постоянном токе и ее аппроксимация с помощью закона Аррениуса. 


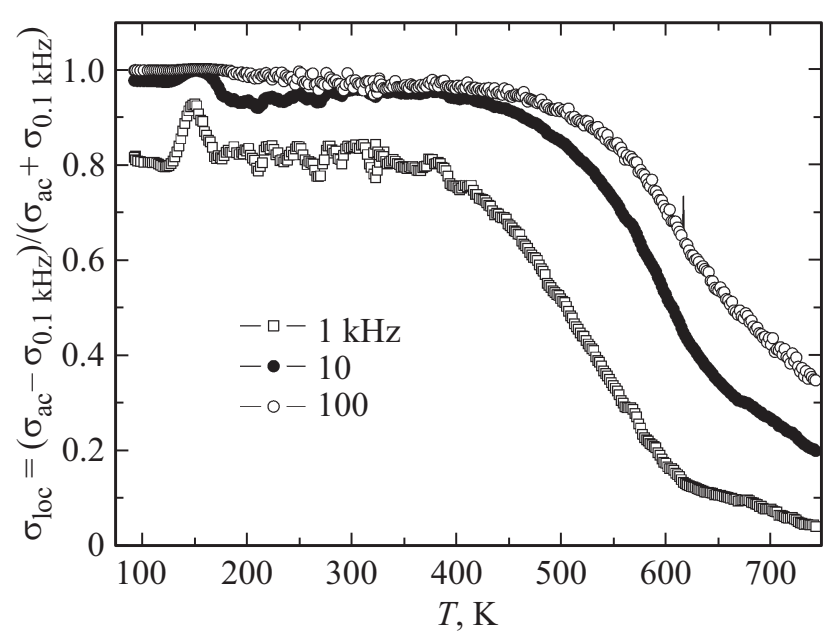

Рис. 3. Температурная зависимость локальной проводимости на разных частотах.

мость. Локальная проводимость существует в широком интервале температур 80-400 K, изменяя свое поведение при варьировании температуры и частоты. Можно выделить две характерные области на температурных зависимостях относительной локальной проводимости: 80-200 K и 200-350 K (рис. 3). В первом интервале температур наблюдается частотно-зависимый максимум локальной проводимости $\sigma_{\mathrm{loc}}$ в окрестности $150 \mathrm{~K}$. Этой аномалии соответствует аномалия диэлектрической проницаемости на рис. 1 и проводимости на рис. 2. Наблюдаемые на рис. 2 и 3 максимумы проводимости при тех же температурах, что и максимумы диэлектрической проницаемости на рис. $1, b$, дают возможность определить активационные барьеры на границах основных локальных областей, ответственных за этот тип аномалии. Из температурно-частотного сдвига положений максимумов проводимости $T_{m}$ (рис. 2,3 ), который описывается законом Аррениуса $\omega=\omega_{0} \cdot \exp \left(-E_{a} / k T_{m}\right)$, (где $\omega_{0}$ - частота попыток перескоков, $E_{a}$ - энергия активации), была получена величина активационного барьера носителей заряда, который составил $770 \mathrm{meV}$. Второй интервал температур (200-350 K) на температурной зависимости локальной проводимости (рис. 3.) характерен отсутствием зависимости $\sigma_{\text {loc }}$ от температуры. При дальнейшем увеличении температуры наблюдается резкое уменьшение локальной проводимости и увеличение сквозной проводимости.

На этом этапе анализа результатов интересно сопоставить наблюдавшуюся картину проводимости с близким по структурному мотиву релаксорным сегнетоэлектриком с магнитоактивным ионом в $B$-подрешетке, соединением $\mathrm{PbCo}_{1 / 3} \mathrm{Nb}_{2 / 3} \mathrm{O}_{3}$ (PCN). Недавние исследования диэлектрических свойств, проводимости, поляризации и структуры PCN [16] показали существование при низких температурах фазового расслоения, обусловленного присутствием в объеме кристалла ионов $\mathrm{Co}^{2+}$ и $\mathrm{Co}^{3+}$. В рассматриваемом здесь соединении PNN также могут присутствовать ионы в различном зарядовом состоянии: $\mathrm{Ni}^{2+}$ и $\mathrm{Ni}^{3+}$, а также $\mathrm{Ni}^{4+}$. Тогда возникновение частотно-зависимой аномалии проводимости в окрестности $150 \mathrm{~K}$ может быть связано с образованием областей с фазовым расслоением. В пользу этого предположения говорит и близость энергии активации в PNN и PCN в окрестности $T_{m}$ (в обоих случаях она равно $\left.E_{a}=770 \mathrm{meV}\right)$. Для выбора корректной модели, описывающей поведение диэлектрических свойств и проводимости, мы продолжим исследования PNN в области гелиевых температур, куда, по-видимому, сместились аномалии проводимости, связанные с формированием областей с зарядовым расслоением.

\section{2. Ток термостимулированной деполяризации}

Поскольку в интервале температур 80-310 К на температурной зависимости dc-проводимости нет заметных аномалий, а ее величина порядка $10^{-12}-10^{-11} \Omega^{-1} \mathrm{~cm}^{-1}$ не должна приводить к заметному экранированию связанного заряда, то с помощью метода термостимулированной деполяризации становится возможным качественно оценить область существования полярного состояния в этом интервале температур. На рис. 4. показана температурная зависимость плотности тока деполяризации при нагревании с постоянной скоростью $V_{T}=5 \mathrm{~K} / \mathrm{min}$, после предварительной поляризации в поле $30 \mathrm{~V}(1.1 \mathrm{kV} / \mathrm{cm})$ разной полярности. Максимумы на температурной зависимости соответствуют максимальному изменению поляризации при изменении температуры согласно выражению

$$
J(T)=\partial P / \partial T \cdot \partial T / \partial t=V_{T} \cdot \partial T / \partial T,
$$

где $V_{T}-$ скорость нагревания.

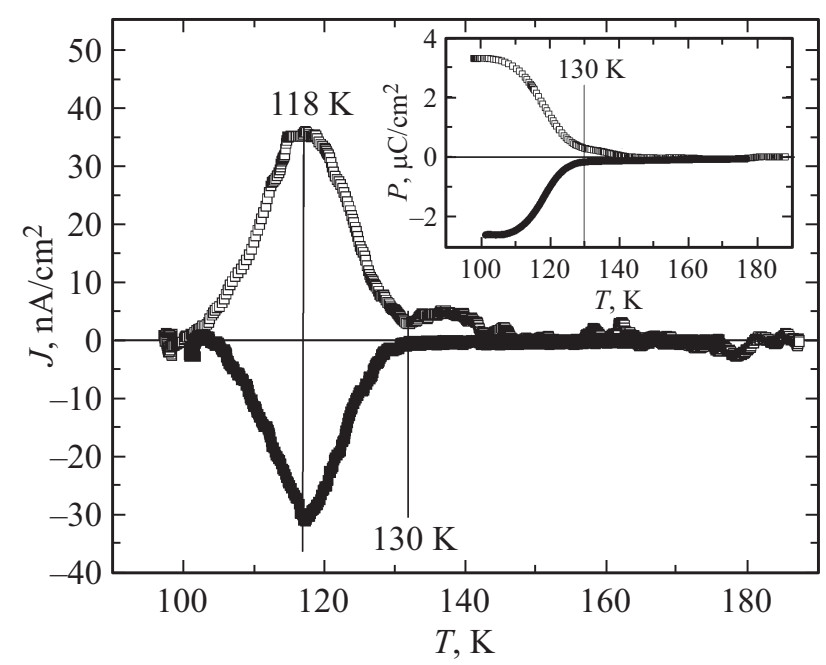

Рис. 4. Ток термостимулированной деполяризации. На вставке - температурная зависимость поляризации, полученная после интегрирования тока. 
Из рисунка видно, что в окрестности $118 \mathrm{~K}$ существует максимум тока деполяризации, а при температуре выше $130 \mathrm{~K}$ его величина падает до нуля. Точно такая же температурная зависимость тока, но другой полярности получена после изменения направления поляризующего поля. Интегрирование плотности тока деполяризации $J(T)$ по времени позволяет качественно представить температурную зависимость величины поляризации $P(T)$ (рис. 4, вставка). На рисунке видно, что величина поляризации ниже $100 \mathrm{~K}$ достигает порядка $2.5-3.0 \mu \mathrm{C} / \mathrm{cm}^{2}$, а с увеличением температуры убывает почти до нуля в окрестности температуры $130 \mathrm{~K}$. Можно отметить, что эта температура на $13 \mathrm{~K}$ ниже области аномалий диэлектрического отклика в окрестности температуры $153 \mathrm{~K}$. Похожая ситуация наблюдается в кристалле PCN, в котором пики тока деполяризации существуют при 180-200 K, полярное состояние исчезает в окрестности $215 \mathrm{~K}$, а максимумы диэлектрической восприимчивости проявляются при более высокой температуре $255 \mathrm{~K}[13,16]$.

\section{4. Заключение}

Аномалии диэлектрического отклика в монокристаллах PNN в виде широких частотно-зависимых максимумов наблюдаются при температурах 153 и $730 \mathrm{~K}$. Получены температурные зависимости проводимости на постоянном и переменном токе, которые имеют одинаковые энергии активации $770 \mathrm{meV}$, что предполагает переход локальной проводимости в объемную при температуре выше $500 \mathrm{~K}$. Результаты исследования тока термостимулированной деполяризации показали, что наведенная поляризация существует в области температур ниже $130 \mathrm{~K}$

\section{Конфликт интересов}

Авторы заявляют, что у них нет конфликта интересов.

\section{Список литературы}

[1] Г.А. Смоленский, В.А. Боков, В.А. Исупов, Н.Н. Крайник, Р.Е. Пасынков, А.И. Соколов, Н.К. Юшин. Физика сегнетоэлектрических явлений. Наука, Л. (1985). 396 с. [G.A. Smolenskii, V.A. Bokov, V.A. Isupov, N.N. Krainik, R.E. Pasynkov, A.I. Sokolov, N.K. Yushin. Ferroelectrics Related Materials. Gordon and Breach Sci. Publ. N.Y.-London (1984). 763 p.]

[2] R. Blinc, P. Cevc, A. Zorko, J. Holc, M. Kosec, Z. Trontelj, J. Pirnat, N. Dalal, V. Ramachandran, J. Krzystek. J. Appl. Phys. 101, 033901 (2007).

[3] N. Ortega, A. Kumar, J.F. Scott, R.S. Katiyar. J. Phys. Condens. Matter 27, 504002 (2015).

[4] В.А. Боков, И.Е. Мыльникова. ФТТ 3, 841 (1961).

[5] И.Е. Мыльникова, В.А. Боков. Кристаллография 4, 433 (1959).

[6] В.А. Боков, И.Е. Мыльникова. ФТТ 11, 2728 (1960).
[7] H.J. Fan, M.H. Kuok, S.C. Ng, N. Yasuda, H. Ohwa, M. Iwata, H. Orihara, Y. Ishibashi. J. Appl. Phys. 91, 4, 2262 (2002).

[8] J. Kano, H. Taniguchi, D. Fu, M. Itoh, S. Kojima. Ferroelectrics 367, 67 (2008).

[9] T.A. Smirnova, A.I. Fedoseev, S.G. Lushnikov, R.S. Katiyar. Ferroelectrics 532, 50 (2018).

[10] R.A. Cowley, S.N. Gvasaliya, S.G. Lushnikov, B. Roessli, G.M. Rotaru. Adv. Phys. 60, 2, 229 (2011).

[11] T. Shirakami, M. Mituskawa, T. Imai, K. Urabe. J. Appl. Phys. 39, Part 2, 7A, L678 (2000).

[12] T. Kimura, T. Goto, H. Shintani, K. Ishizaka, T. Arima, Y. Tokura. Nature 426, 55 (2003).

[13] V. Zalesskii, T. Smirnova, S. Lushnikov. Ferroelectrics 538, 153 (2019)

[14] A.D. Polushina, E.D. Obozova, T.A. Smirnova, V.G. Zalesskii, S.G. Lushnikov. J. Phys.: Conf. Ser. 1400, 077003 (2019).

[15] A.R. Long. Adv. Phys. 31, 587 (1982).

[16] Б.Х. Хананов, В.Г. Залесский, Е.И. Головенчиц, В.А. Санина, Т.А. Смирнова, М.П. Щеглов, В.А. Боков, С.Г. Лушников. ЖЭТФ 157, 3, 523 (2020).

Редактор Д.В. Жуманов 